\title{
Asymptotic Tracking for Systems With Structured and Unstructured Uncertainties
}

\author{
P. M. Patre, Student Member, IEEE, W. MacKunis, C. Makkar, and W. E. Dixon, Senior Member, IEEE
}

\begin{abstract}
The control of systems with uncertain nonlinear dynamics has been a decades-long mainstream area of focus. The general trend for previous control strategies developed for uncertain nonlinear systems is that the more unstructured the system uncertainty, the more control effort (i.e., high gain or high-frequency feedback) is required to cope with the uncertainty, and the resulting stability and performance of the system is diminished (e.g., uniformly ultimately bounded stability). This brief illustrates how the amalgamation of an adaptive model-based feedforward term (for linearly parameterized uncertainty) with a robust integral of the sign of the error (RISE) feedback term (for additive bounded disturbances) can be used to yield an asymptotic tracking result for Euler-Lagrange systems that have mixed unstructured and structured uncertainty. Experimental results are provided that illustrate a reduced root-mean-squared tracking error with reduced control effort.
\end{abstract}

Index Terms-Adaptive control, friction, Lyapunov methods, nonlinearities, robustness.

\section{INTRODUCTION}

$\mathbf{T}$ HE control of systems with uncertain nonlinear dynamics has been a decades-long mainstream area of focus. For systems with uncertainties that can be linearly parameterized, a variety of adaptive (e.g., [8], [21], [23]) feedforward controllers can be utilized to achieve an asymptotic result. Some recent adaptive control results have also targeted the application of adaptive controllers for systems that are not linear in the parameters [11]. Learning controllers have been developed for systems with periodic disturbances [1], and recent research has focused on the use of exosystems [22] to compensate for disturbances that are the solution of a linear time-invariant system with unknown coefficients. A variety of methods have also been proposed to compensate for systems with unstructured uncertainty including: various sliding mode controllers (e.g., [23], [24]), robust control schemes [20], and neural network and fuzzy logic controllers (e.g., [9], [10], and [19]). From a review of these approaches, a general trend that can be determined is that controllers developed for systems with more unstructured

Manuscript received February 28, 2007; revised May 7, 2007. Manuscript received in final form June 28, 2007. Recommended by Associate Editor Y. Jin. This work was supported in part by the National Science Foundation through CAREER Award CMS-0547448, the Air Force Office of Scientific Research under Contract F49620-03-1-0381, Contract F49620-03-1-0170, and Contract FA4819-05-D-0011, BARD under Grant US-3715-05, and by the U.S.-Israel Binational Agricultural Research and Development Fund at the University of Florida.

The authors are with the Department of Mechanical and Aerospace Engineering University of Florida, Gainesville, FL 32611-6250 USA (e-mail: pmpatre@ufl.edu; mackunis@ufl.edu; cmakkar@ufl.edu; wdixon@ufl.edu).

Color versions of one or more of the figures in this paper are available online at http://ieeexplore.ieee.org.

Digital Object Identifier 10.1109/TCST.2007.908227 uncertainty will require more control effort (i.e., high gain or high-frequency feedback) and yield reduced performance (e.g., uniformly ultimately bounded stability).

Recently, a new high gain feedback control strategy called the robust integral of the sign of the error (RISE) was developed in [25] that contains a unique integral signum term which can accommodate for sufficiently smooth bounded disturbances. A significant outcome of this new control structure is that asymptotic stability is obtained despite a fairly general uncertain disturbance. This technique was used in [2] to develop a tracking controller for nonlinear systems in the presence of additive disturbances and parametric uncertainties under the assumption that the disturbances are $C^{2}$ with bounded time derivatives. In [27], Xian et al. utilized this strategy to propose a new output feedback discontinuous tracking controller for a general class of nonlinear mechanical (i.e., second-order) systems whose uncertain dynamics are first-order differentiable. In [28], Zhang et al. combined the RISE feedback structure with a high-gain observer at the sacrifice of yielding a semi-global uniformly ultimately bounded result. The RISE method has also been used as an identification technique. For example, the method has been applied to identify friction (e.g., [14] and [15]), for range identification in perspective and paracatadioptric vision systems (e.g., [5] and [6]), and for fault detection and identification (e.g., [17]).

The development in this brief is motivated by the desire to include some knowledge of the dynamics in the control design as a means to improve the performance and reduce the control effort. Specifically, for systems that include some dynamics that can be segregated into structured (i.e., linear parameterizable) and unstructured uncertainty, this brief illustrates how a new controller and error system can be crafted to include a model-based adaptive feedforward term in conjunction with the RISE feedback technique to yield an asymptotic tracking result. This brief presents the first result that illustrates how the amalgamation of these compensation methods can be used to yield an asymptotic result. Heuristically, the addition of the model-based adaptive feedforward term should reduce the overall control effort because some of the disturbance has been isolated and compensated for by a non-high-gain feedforward element. Moreover, the addition of the adaptive feedforward term injects some knowledge of the dynamics in the control structure, which could lead to improved performance. Experimental results are presented to reinforce these heuristic notions. Specifically, the presented controller was implemented on a simple one-link robot testbed and demonstrated reduced tracking error and control effort. For this testbed, the dynamics that were included in the feedforward term included the inertia of the linkage assembly, and the friction present in the system. 


\section{DYNAMIC MODEL AND PROPERTIES}

The class of nonlinear dynamic systems considered in this brief is assumed to be modeled by the following Euler-Lagrange formulation:

$$
M(q) \ddot{q}+V_{m}(q, \dot{q}) \dot{q}+G(q)+f(\dot{q})+\tau_{d}(t)=\tau(t)
$$

In (1), $M(q) \in \mathbb{R}^{n \times n}$ denotes the inertia matrix, $V_{m}(q, \dot{q}) \in$ $\mathbb{R}^{n \times n}$ denotes the centripetal-Coriolis matrix, $G(q) \in \mathbb{R}^{n}$ denotes the gravity vector, $f(\dot{q}) \in \mathbb{R}^{n}$ denotes friction, $\tau_{d}(t) \in \mathbb{R}^{n}$ denotes a general nonlinear disturbance (e.g., unmodeled effects), $\tau(t) \in \mathbb{R}^{n}$ represents the torque input control vector, and $q(t), \dot{q}(t), \ddot{q}(t) \in \mathbb{R}^{n}$ denote the link position, velocity, and acceleration vectors, respectively. The friction term $f(\dot{q})$ in (1) is assumed to have the following form as in [16]:

$$
f(\dot{q})=\gamma_{1}\left(\tanh \left(\gamma_{2} \dot{q}\right)-\tanh \left(\gamma_{3} \dot{q}\right)\right)+\gamma_{4} \tanh \left(\gamma_{5} \dot{q}\right)+\gamma_{6} \dot{q}
$$

where $\gamma_{i} \in \mathbb{R} \forall i=1,2, \ldots, 6$, denote unknown positive constants. The friction model in (2) has the following properties: 1) it is symmetric about the origin; 2) it has a static coefficient of friction; 3) it exhibits the Stribeck effect where the friction coefficient decreases from the static coefficient of friction with increasing slip velocity near the origin; 4) it includes a viscous dissipation term; and 5) it has a Coulombic friction coefficient in the absence of viscous dissipation. To a good approximation, the static friction coefficient is given by $\gamma_{1}+\gamma_{4}$, and the Stribeck effect is captured by $\tanh \left(\gamma_{2} \dot{q}\right)-\tanh \left(\gamma_{3} \dot{q}\right)$. The Coulombic friction coefficient is given by $\gamma_{4} \tanh \left(\gamma_{5} \dot{q}\right)$, and the viscous dissipation is given by $\gamma_{6} \dot{q}$. For further details regarding the friction model, see [16]. The subsequent development is based on the assumption that $q(t)$ and $\dot{q}(t)$ are measurable and that $M(q)$, $V_{m}(q, \dot{q}), G(q), f(\dot{q})$, and $\tau_{d}(t)$ are unknown. Moreover, the following properties and assumptions will be exploited in the subsequent development.

Property 1: The inertia matrix $M(q)$ is symmetric and positive definite and satisfies the following inequality $\forall y(t) \in \mathbb{R}^{n}$ :

$$
m_{1}\|y\|^{2} \leq y^{T} M(q) y \leq \bar{m}(q)\|y\|^{2}
$$

where $m_{1} \in \mathbb{R}$ is a known positive constant, $\bar{m}(q) \in \mathbb{R}$ is a known positive function, and $\|\cdot\|$ denotes the standard Euclidean norm.

Property 2: If $q(t), \dot{q}(t) \in \mathcal{L}_{\infty}$, then the first and second partial derivatives of the elements of $M(q), V_{m}(q, \dot{q})$, and $G(q)$ with respect to $q$, and the first and second partial derivatives of the elements of $V_{m}(q, \dot{q})$, and $f(\dot{q})$ with respect to $\dot{q}$ exist and are bounded.

Property 3: The nonlinear disturbance term and its first two time derivatives are bounded (i.e., $\left.\tau_{d}(t), \dot{\tau}_{d}(t), \ddot{\tau}_{d}(t) \in \mathcal{L}_{\infty}\right)$.

\section{ERROR SYSTEM DEVELOPMENT}

The control objective is to ensure that the system tracks a desired time-varying trajectory despite structured and unstructured uncertainties in the dynamic model. To quantify this objective, a position tracking error, denoted by $e_{1}(t) \in \mathbb{R}^{n}$, is defined as

$$
e_{1} \triangleq q_{d}-q
$$

To facilitate the subsequent analysis, filtered tracking errors, denoted by $e_{2}(t), r(t) \in \mathbb{R}^{n}$, are also defined as

$$
\begin{array}{r}
e_{2} \triangleq \dot{e}_{1}+\alpha_{1} e_{1} \\
r \triangleq \dot{e}_{2}+\alpha_{2} e_{2}
\end{array}
$$

where $\alpha_{1}, \alpha_{2} \in \mathbb{R}$ denote positive constants. The filtered tracking error $r(t)$ is not measurable since the expression in (6) depends on $\ddot{q}(t)$.

The open-loop tracking error system can be developed by premultiplying (6) by $M(q)$ and utilizing the expressions in (1), (2), (4), and (5) to obtain the following expression:

$$
M(q) r=Y_{d} \theta+S+W_{d}+\tau_{d}(t)-\tau(t)
$$

where $Y_{d}\left(q_{d}, \dot{q}_{d}, \ddot{q}_{d}\right) \theta \in \mathbb{R}^{n}$ is defined as

$$
\begin{aligned}
Y_{d} \theta \triangleq & M\left(q_{d}\right) \ddot{q}_{d}+V_{m}\left(q_{d}, \dot{q}_{d}\right) \dot{q}_{d}+G\left(q_{d}\right) \\
& +\gamma_{1}\left(\tanh \left(\bar{\gamma}_{2} \dot{q}_{d}\right)-\tanh \left(\bar{\gamma}_{3} \dot{q}_{d}\right)\right) \\
& +\gamma_{4} \tanh \left(\bar{\gamma}_{5} \dot{q}_{d}\right)+\gamma_{6} \dot{q}_{d} .
\end{aligned}
$$

In (8), $\theta \in \mathbb{R}^{p}$ contains the constant unknown system parameters, $Y_{d}\left(q_{d}, \dot{q}_{d}, \ddot{q}_{d}\right) \in \mathbb{R}^{n \times p}$ is the desired regression matrix that contains known functions of the desired link position, velocity, and acceleration, $q_{d}(t), \dot{q}_{d}(t), \ddot{q}_{d}(t) \in \mathbb{R}^{n}$, respectively, and $\bar{\gamma}_{2}, \bar{\gamma}_{3}, \bar{\gamma}_{5} \in \mathbb{R}$ are the best guess estimates for $\gamma_{2}, \gamma_{3}$, and $\gamma_{5}$, respectively. The desired trajectory is assumed to be designed such that $q_{d}(t), \dot{q}_{d}(t), \ddot{q}_{d}(t), \dddot{q}_{d}(t), \dddot{q}_{d}(t) \in \mathbb{R}^{n}$ exist and are bounded. In (7), the auxiliary function $S\left(q, \dot{q}, q_{d}, \dot{q}_{d}, \ddot{q}_{d}\right) \in \mathbb{R}^{n}$ is defined as

$$
\begin{aligned}
S \triangleq & M(q)\left(\alpha_{1} \dot{e}_{1}+\alpha_{2} e_{2}\right)+M(q) \ddot{q}_{d}-M\left(q_{d}\right) \ddot{q}_{d} \\
& +V_{m}(q, \dot{q}) \dot{q}-V_{m}\left(q_{d}, \dot{q}_{d}\right) \dot{q}_{d}+G(q)-G\left(q_{d}\right)+\gamma_{6} \dot{q} \\
& -\gamma_{6} \dot{q}_{d}+\gamma_{4} \tanh \left(\gamma_{5} \dot{q}\right)-\gamma_{4} \tanh \left(\gamma_{5} \dot{q}_{d}\right) \\
& +\gamma_{1}\left(\tanh \left(\gamma_{2} \dot{q}\right)-\tanh \left(\gamma_{3} \dot{q}\right)\right) \\
& -\gamma_{1}\left(\tanh \left(\gamma_{2} \dot{q}_{d}\right)-\tanh \left(\gamma_{3} \dot{q}_{d}\right)\right)
\end{aligned}
$$

and the auxiliary function $W_{d}\left(\dot{q}_{d}\right) \in \mathbb{R}^{n}$ is defined as

$$
\begin{aligned}
W_{d} \triangleq & \gamma_{4} \tanh \left(\gamma_{5} \dot{q}_{d}\right)-\gamma_{4} \tanh \left(\bar{\gamma}_{5} \dot{q}_{d}\right) \\
& +\gamma_{1}\left(\tanh \left(\gamma_{2} \dot{q}_{d}\right)-\tanh \left(\gamma_{3} \dot{q}_{d}\right)\right) \\
& -\gamma_{1}\left(\tanh \left(\bar{\gamma}_{2} \dot{q}_{d}\right)-\tanh \left(\bar{\gamma}_{3} \dot{q}_{d}\right)\right)
\end{aligned}
$$

Based on the expression in (7), the control torque input is designed as follows:

$$
\tau=Y_{d} \hat{\theta}+\mu
$$


In (11), $\mu(t) \in \mathbb{R}^{n}$ denotes the RISE term defined as

$$
\begin{aligned}
\mu(t) \triangleq & \left(k_{s}+1\right) e_{2}(t)-\left(k_{s}+1\right) e_{2}(0) \\
& +\int_{0}^{t}\left[\left(k_{s}+1\right) \alpha_{2} e_{2}(\sigma)+\beta s g n\left(e_{2}(\sigma)\right)\right] d \sigma
\end{aligned}
$$

where $k_{s}, \beta \in \mathbb{R}$ are positive, constant control gains, and $\hat{\theta}(t) \in$ $\mathbb{R}^{p}$ denotes a parameter estimate vector generated online according to the following update law:

$$
\dot{\hat{\theta}}=\Gamma \dot{Y}_{d}^{T} r
$$

where $\Gamma \in \mathbb{R}^{p \times p}$ is a known, constant, diagonal, positive-definite adaptation gain matrix. Since $\dot{Y}_{d}(t)$ is only a function of the known desired time varying trajectory, (13) can be integrated by parts as follows:

$\hat{\theta}(t)=\hat{\theta}(0)+\left.\Gamma \dot{Y}_{d}^{T} e_{2}(\sigma)\right|_{0} ^{t}-\Gamma \int_{0}^{t}\left\{\ddot{Y}_{d}^{T} e_{2}(\sigma)-\alpha_{2} \dot{Y}_{d}^{T} e_{2}(\sigma)\right\} d \sigma$

so that the parameter estimate vector $\hat{\theta}(t)$ implemented in (11) does not depend on the unmeasurable signal $r(t)$.

Remark 1: The control design in (11) is similar to the results in [25]. However, previous designs based on [25] could only compensate for uncertainty in the system through the high-gain RISE feedback term $\mu(t)$. Through the new development presented in the current result, an adaptive feedforward term can also be used to compensate for system uncertainty. This flexibility presents a significant advantage because it allows more system dynamics to be incorporated in the control design. Specifically, if some of the system uncertainty can be segregated into a linear parameterizable form, then the model-based adaptive feedforward term can be injected to compensate for the uncertainty instead of just relying on the non-model based high gain RISE feedback term. Heuristically, this contribution should improve the tracking performance and reduce the control effort. Experimental results on a simple one-link robot manipulator provide some validation of this heuristic idea.

The closed-loop tracking error system can be developed by substituting (11) into (7) as

$$
M(q) r=Y_{d} \tilde{\theta}+S+W_{d}+\tau_{d}-\mu(t)
$$

where $\tilde{\theta}(t) \in \mathbb{R}^{p}$ represents the parameter estimation error vector defined as

$$
\tilde{\theta} \triangleq \theta-\hat{\theta}
$$

To facilitate the subsequent stability analysis (and to illustrate some insight into the structure of the design for $\mu(t)$ ), the time derivative of (15) is determined as

$$
M(q) \dot{r}=-\frac{1}{2} \dot{M}(q) r+\dot{Y}_{d} \tilde{\theta}+\tilde{N}(t)+N_{d}(t)-\dot{\mu}(t)-e_{2}
$$

where the unmeasurable auxiliary term $\tilde{N}\left(e_{1}, e_{2}, r\right) \in \mathbb{R}^{n}$ is defined as

$$
\tilde{N}(t) \triangleq-Y_{d} \Gamma \dot{Y}_{d}^{T} r+\dot{S}-\frac{1}{2} \dot{M}(q) r+e_{2}
$$

where (13) was used. In (17), the unmeasurable auxiliary term $N_{d}\left(q_{d}, \dot{q}_{d}, \ddot{q}_{d}\right) \in \mathbb{R}^{n}$ is defined as

$$
N_{d}(t) \triangleq \dot{W}_{d}+\dot{\tau}_{d}
$$

The time derivative of (12) is given as

$$
\dot{\mu}(t)=\left(k_{s}+1\right) r+\beta \operatorname{sgn}\left(e_{2}\right) .
$$

The expressions in (12) and (20) are based on [25]. In a similar manner as in [26], the Mean Value Theorem can be used to develop the following upper bound:

$$
\|\tilde{N}(t)\| \leq \rho(\|z\|)\|z\|
$$

where $z(t) \in \mathbb{R}^{3 n}$ is defined as

$$
z(t) \triangleq\left[\begin{array}{lll}
e_{1}^{T} & e_{2}^{T} & r^{T}
\end{array}\right]^{T}
$$

The following inequalities can be developed based on the expression in (19) and its time derivative:

$$
\left\|N_{d}(t)\right\| \leq \zeta_{N_{d}} \quad\left\|\dot{N}_{d}(t)\right\| \leq \zeta_{N_{d 2}}
$$

where $\zeta_{N_{d}}, \zeta_{N_{d 2}} \in \mathbb{R}$ are known positive constants.

\section{Stability ANALYSIS}

Theorem: The controller given in (11), (12), and (14) ensures that all system signals are bounded under closed-loop operation and that the position tracking error is regulated in the sense that

$$
\left\|e_{1}(t)\right\| \rightarrow 0 \quad \text { as } \quad t \rightarrow \infty
$$

provided the control gain $k_{s}$ introduced in (12) is selected sufficiently large, and $\alpha_{1}$ and $\alpha_{2}$ are selected according to the following sufficient condition:

$$
\alpha_{1}>\frac{1}{2} ; \quad \alpha_{2}>1
$$

and $\beta$ is selected according to the following sufficient condition:

$$
\beta>\zeta_{N_{d}}+\frac{1}{\alpha_{2}} \zeta_{N_{d 2}}
$$

where $\zeta_{N_{d}}$ and $\zeta_{N_{d 2}}$ are introduced in (23). [18].

Proof: For details of the proof, please see [4], [13], and

\section{EXPERIMENTAL RESULTS}

The testbed depicted in Fig. 1 was used to implement the developed controller. The testbed consists of a circular disc of unknown inertia mounted on an NSK direct-drive switched reluctance motor (240.0 Nm Model YS5240-GN001). The 


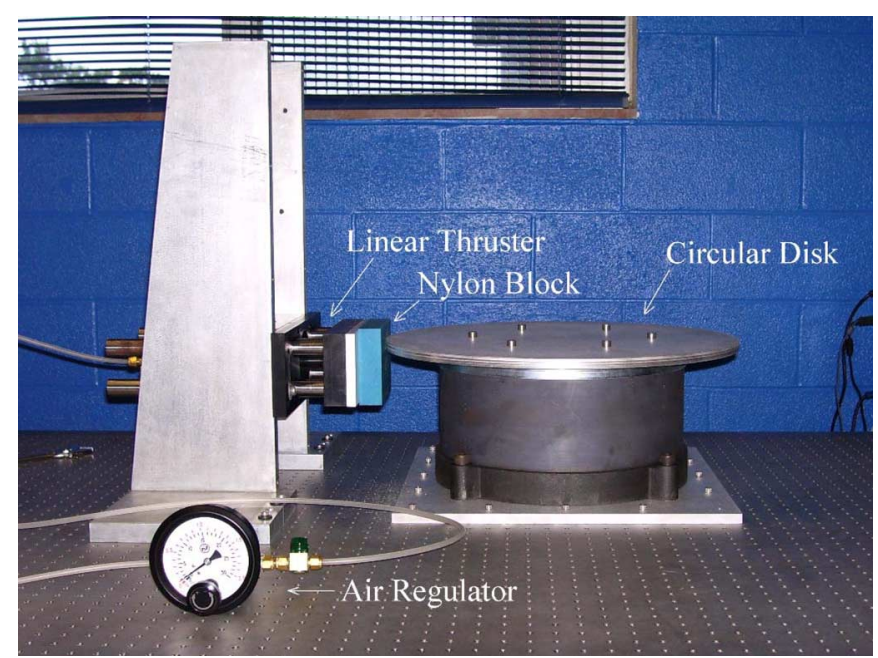

Fig. 1. Experimental testbed consists of a circular disk mounted on an NSK direct-drive switched reluctance motor.

NSK motor is controlled through power electronics operating in torque control mode. The motor resolver provides rotor position measurements with a resolution of $614400 \mathrm{pulses} / \mathrm{rev}$ olution. A Pentium 2.8-GHz PC operating under QNX hosts the control algorithm, which was implemented via Qmotor 3.0, a graphical user-interface, to facilitate real-time graphing, data logging, and adjustment of control gains without recompiling the program (for further information on Qmotor 3.0, the reader is referred to [12]). Data acquisition and control implementation were performed at a frequency of $1.0 \mathrm{kHz}$ using the ServoToGo I/O board. A rectangular nylon block was mounted on a pneumatic linear thruster to apply an external friction load to the rotating disk. A pneumatic regulator maintained a constant pressure of 20 pounds per square inch on the circular disk.

The dynamics for the testbed are given as follows:

$$
J \ddot{q}+f(\dot{q})+\tau_{d}(t)=\tau(t)
$$

where $J \in \mathbb{R}$ denotes the combined inertia of the circular disk and rotor assembly, the friction torque $f(\dot{q}) \in \mathbb{R}$ is defined in (2), and $\tau_{d}(t) \in \mathbb{R}$ denotes a general nonlinear disturbance (e.g., unmodeled effects). The control torque input $\tau(t)$ is given by (11), where $Y_{d}\left(\dot{q}_{d}, \ddot{q}_{d}\right) \in \mathbb{R}^{1 \times 4}$ is the regression matrix defined as $Y_{d} \triangleq\left[\begin{array}{lll}\ddot{q}_{d} & \tanh \left(\bar{\gamma}_{2} \dot{q}_{d}\right)-\tanh \left(\bar{\gamma}_{3} \dot{q}_{d}\right) \quad \tanh \left(\bar{\gamma}_{5} \dot{q}_{d}\right) \quad \dot{q}_{d}\end{array}\right]$

and $\hat{\theta}(t) \in \mathbb{R}^{4}$ is the vector consisting of the unknown parameters defined as

$$
\hat{\theta} \triangleq\left[\begin{array}{llll}
\hat{J} & \hat{\gamma}_{1} & \hat{\gamma}_{4} & \hat{\gamma}_{6}
\end{array}\right]^{T}
$$

The parameter estimates vector in (27) is generated online using the adaptive update law in (14). The desired link trajectory (see Fig. 2 was selected as follows (in degrees):

$$
q_{d}(t)=45.0 \sin (1.2 t)\left(1-\exp \left(-0.01 t^{3}\right)\right) .
$$

For all experiments, the rotor velocity signal is obtained by applying a standard backwards difference algorithm to the position signal. The integral structure of the adaptive term in (14) and the RISE term in (12) was computed online via a standard

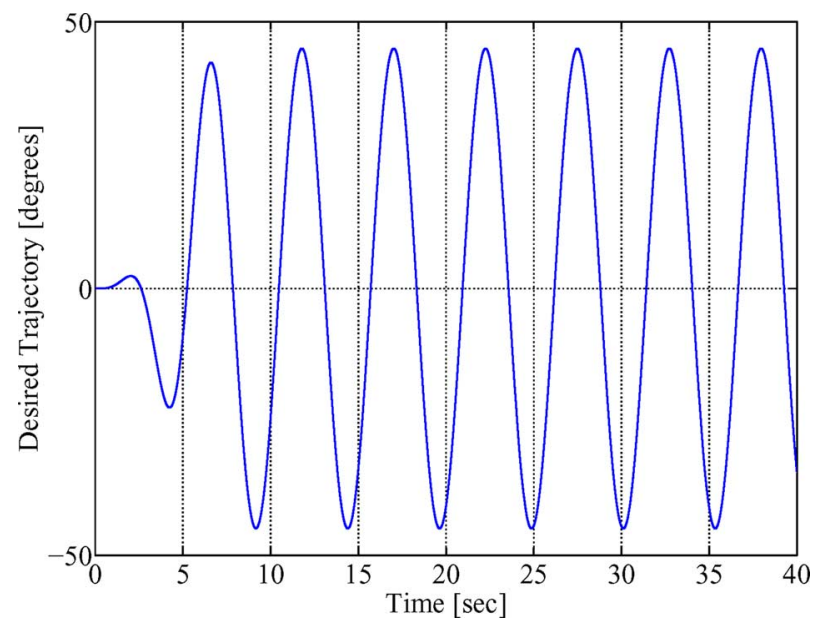

Fig. 2. Desired trajectory used for the experiment.

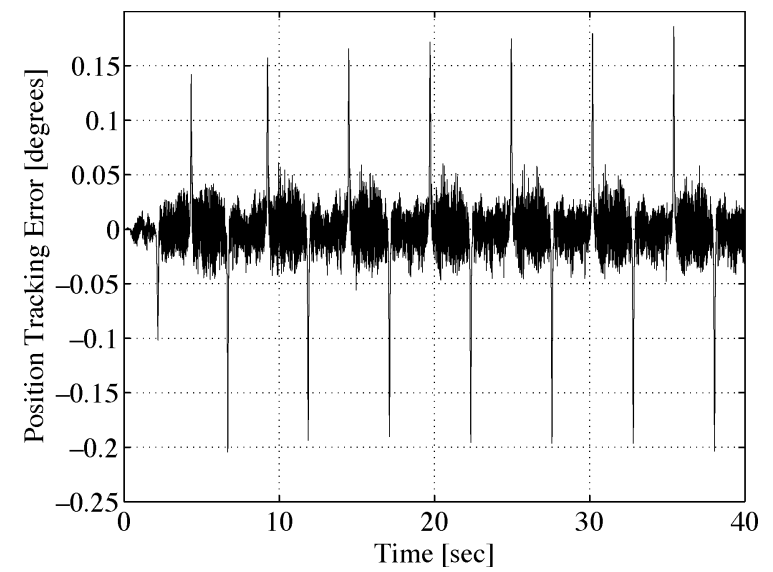

Fig. 3. Position tracking error without the adaptive feedforward term.

trapezoidal algorithm. In addition, all the states and unknown parameters were initialized to zero. The signum function for the control scheme in (12) was defined as

$$
\operatorname{sgn}\left(e_{2}(t)\right)= \begin{cases}1, & e_{2}>0.0005 \\ 0, & -0.0005<e_{2} \leq 0.0005 \\ -1, & e_{2} \leq-0.0005\end{cases}
$$

Experiment 1: In the first experiment, the controller in (11) was implemented without including the adaptation term. Thus, the control torque input given in (11) takes the following form [25]:

$$
\tau(t)=\mu(t)
$$

The gains for the controller that yielded the best steady-state performance were determined as follows:

$$
k_{s}=100 \quad \beta=115 \quad \alpha_{1}=40 \quad \alpha_{2}=30 .
$$

The position tracking error obtained from the controller is plotted in Fig. 3, and the torque input by the controller is depicted in Fig. 4. 


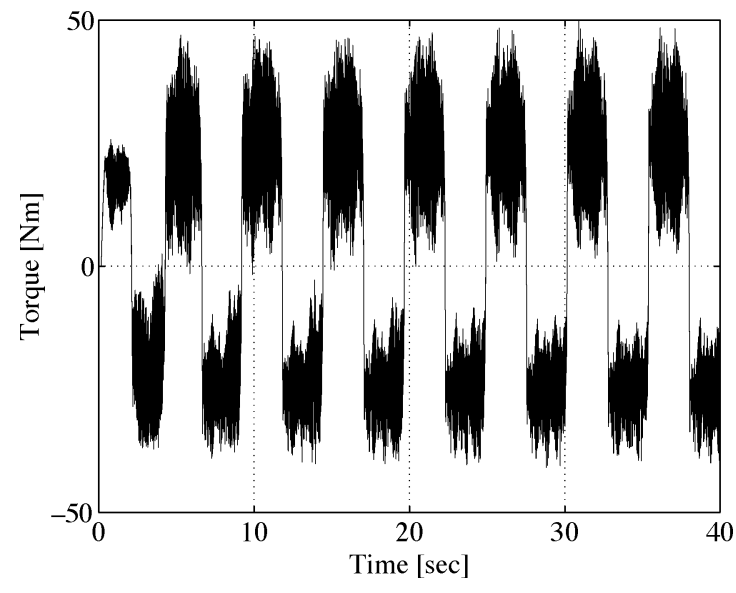

Fig. 4. Torque input without the adaptive feedforward term.

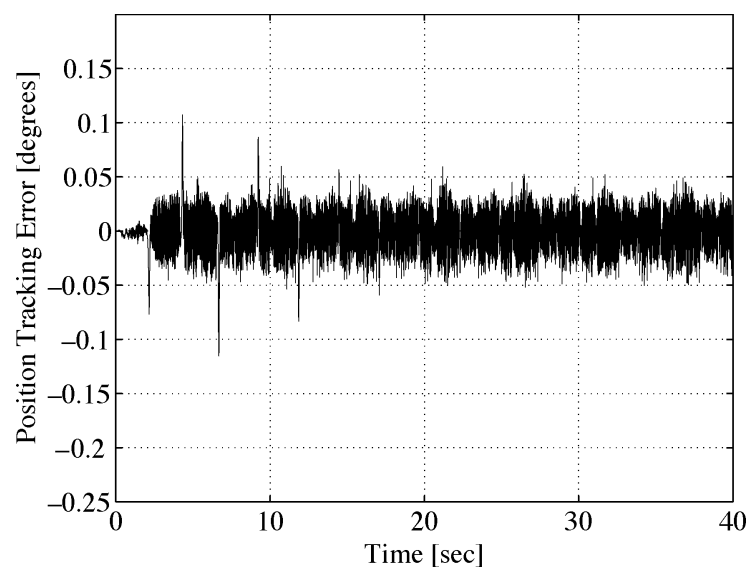

Fig. 5. Position tracking error for the control structure that includes the adaptive feedforward term.

Experiment 2: In the second experiment, the control input given in (11) was used. The update law defined in (14) was used to update the parameter estimates defined in (27). The following control gains and best guess estimates were used to implement the controller in (11):

$$
\begin{aligned}
k_{s} & =100 \\
\beta & =115 \\
\alpha_{1} & =40 \\
\alpha_{2} & =30 \\
\Gamma & =\operatorname{diag}\{10,1,1,10\} \\
\bar{\gamma}_{2} & =50 \\
\bar{\gamma}_{3} & =1 \\
\bar{\gamma}_{5} & =50 .
\end{aligned}
$$

The position tracking error obtained from the controller is plotted in Fig. 5, the parameter estimates are depicted in Fig. 6, the contribution of the RISE term is shown in Fig. 8, and the torque input by the controller is depicted in Fig. 7.

\section{DISCUSSION}

Fig. 5 illustrates that the incorporation of a model-based feedforward term eliminates the spikes present in Fig. 3 that

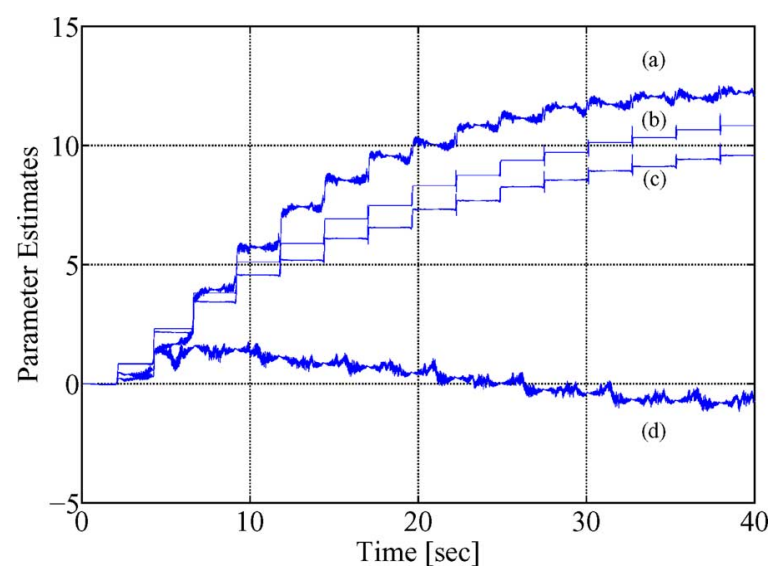

Fig. 6. Parameter estimates of the adaptive feedforward component: (a) $\hat{\gamma}_{1}$ (b) $\hat{\gamma}_{4}$, (c) $\hat{\gamma}_{6}$, and (d) $\hat{J}$.

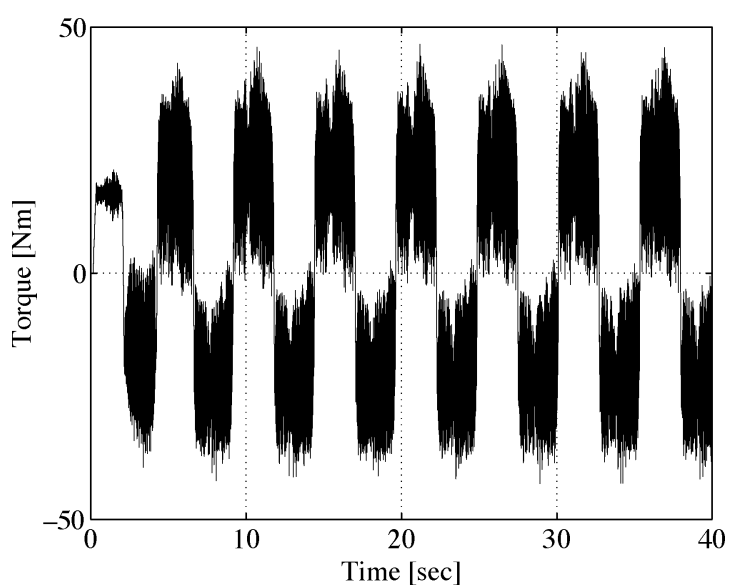

Fig. 7. Torque input for the control structure that includes the adaptive feedforward term.

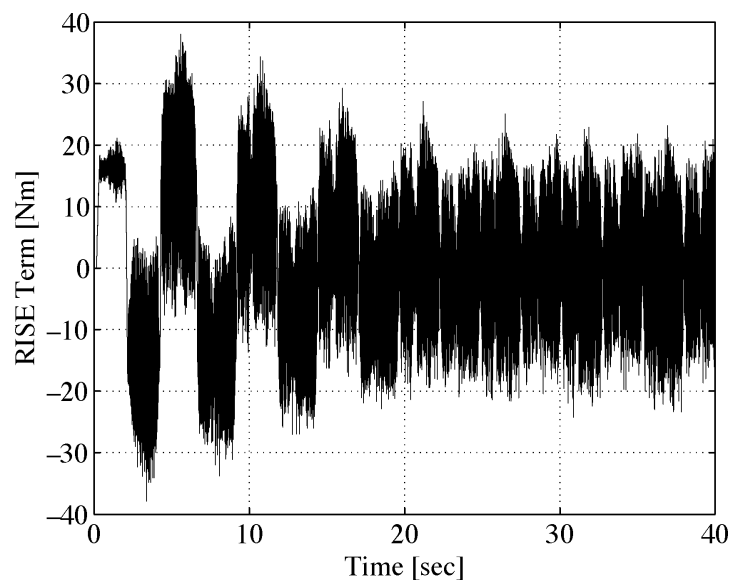

Fig. 8. Contribution of the RISE term for the control structure that includes the adaptive feedforward term.

occur when the motor changes direction. The spikes are initially present in Fig. 5, but reduce in magnitude and vanish as the adaptive update converges. These figures exactly illustrate how the addition of the adaptive feedforward element injects model knowledge into the control design to improve the overall performance. Fig. 8 indicates that the contribution of the RISE 
RMS Error [degrees]

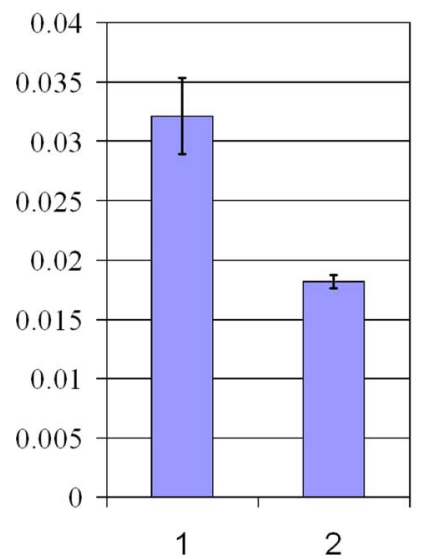

RMS Torque $[\mathrm{N}-\mathrm{m}]$

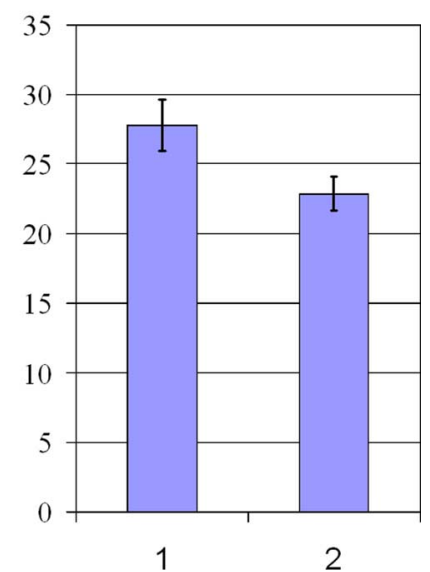

Fig. 9. RMS position tracking errors and torques for the two cases-(1) without the adaptation term in the control input and (2) with the adaptation term in the control input.

TABLE I

T-Test: Two SAMPLES ASSUMing EQUAL VARIANCES FOR RMS ERROR

\begin{tabular}{|l|c|c|}
\hline \multirow{2}{*}{} & \multicolumn{2}{|c|}{ RMS Error } \\
\cline { 2 - 3 } & Variable 1 & Variable 2 \\
\hline Mean & $3.214 \times 10^{-2}$ & $1.817 \times 10^{-2}$ \\
\hline Oariance & $1.044 \times 10^{-5}$ & $3.170 \times 10^{-7}$ \\
\hline Pooservations & 10 & 10 \\
\hline $\begin{array}{l}\text { Hypothesized Mean } \\
\text { Difference }\end{array}$ & $5.378 \times 10^{-6}$ & \\
\hline df & 0 & \\
\hline $\mathrm{t}$ Stat & 18 & \\
\hline $\mathrm{P}(\mathrm{T}<=\mathrm{t})$ one-tail & $3.847 \times 10^{-11}$ & \\
\hline $\mathrm{t}$ Critical one-tail & 1.734 & \\
\hline $\mathrm{P}(\mathrm{T}<=\mathrm{t})$ two-tail & $7.693 \times 10^{-11}$ & \\
\hline $\mathrm{t}$ Critical two-tail & 2.101 & \\
\hline
\end{tabular}

term in the overall torque decreases with time as the feedforward adaptation term begins to compensate for part of the disturbances.

Both of the experiments were repeated ten consecutive times with the same gain values to check the repeatability and accuracy of the results. For each run, the root-mean-squared (rms) values of the position tracking errors and torques are calculated. The average of these rms values for the two cases (with adaptation and without adaptation) obtained over ten sets are plotted in Fig. 9, where the bars indicate the variance about the mean. An unpaired $\mathrm{t}$-test assuming equal variances was performed using a statistical package (Microsoft Office Excel 2003) with a significance level of $\alpha=0.05$. The results of the t-test for the rms error, and the rms torque are shown in Tables I and II, respectively. Table I indicates that the $P$ value obtained for the one-tailed test is less than the significance level $\alpha$. Thus, the mean rms error for case 2 is lower than that of case 1 , and this difference is statistically significant. Similarly, from Table II, the mean rms torque for case 2 is lower than that of case 1 . The results indicate that the mean rms value of the position tracking error when the adaptive feedforward term is used is about $43.5 \%$ less than the case when no adaptation term is used. This improvement in performance by the proposed controller was obtained while using 17.6\% less input torque as shown in Fig. 9.
TABLE II

T-Test: Two SAMPLES ASSUMING EQUAL VARIANCES FOR RMS TORQUE

\begin{tabular}{|l|c|c|}
\hline \multirow{2}{*}{} & \multicolumn{2}{|c|}{ RMS Torque } \\
\cline { 2 - 3 } & Variable 1 & Variable 2 \\
\hline Mean & 27.79 & 22.90 \\
\hline Variance & 3.452 & 1.474 \\
\hline Observations & 10 & 10 \\
\hline Pooled Variance & 2.463 & \\
\hline $\begin{array}{l}\text { Hypothesized Mean } \\
\text { Difference }\end{array}$ & 0 & \\
\hline df & 18 & \\
\hline t Stat & 3.971 & \\
\hline $\mathrm{P}(\mathrm{T}<=\mathrm{t})$ one-tail & $8.208 \times 10^{-7}$ & \\
\hline t Critical one-tail & 1.734 & \\
\hline $\mathrm{P}(\mathrm{T}<=\mathrm{t})$ two-tail & $1.642 \times 10^{-6}$ & \\
\hline t Critical two-tail & 2.101 & \\
\hline
\end{tabular}

\section{CONCLUSION}

A new class of asymptotic controllers is developed that contains an adaptive feedforward term to account for linear parameterizable uncertainty and a high-gain RISE feedback term which accounts for unstructured disturbances. In comparison with previous results that used a similar high-gain feedback control structure, new control development was required to include the additional adaptive feedforward term. The motivation for injecting the adaptive feedforward term is that improved tracking performance and reduced control effort result from including more knowledge of the system dynamics in the control structure. This heuristic idea was verified by our experimental results that indicate reduced control effort and reduced rms tracking errors.

\section{REFERENCES}

[1] R. J. Antsaklis and K. M. Passino, Eds., An Introduction to Intelligent and Autonomous Control. Boston, MA: Kluwer, 1993.

[2] Z. Cai, M. S. de Queiroz, and D. M. Dawson, "Robust adaptive asymptotic tracking of nonlinear systems with additive disturbance," in Proc. IEEE Conf. Decision Control/Eur. Control Conf., Spain, 2005, pp. 2877-2882.

[3] Z. Cai, M. S. de Queiroz, and D. M. Dawson, "Robust adaptive asymptotic tracking of nonlinear systems with additive disturbance," IEEE Trans. Autom. Control, vol. 51, no. 3, pp. 524-529, Mar. 2006.

[4] Z. Cai, M. S. de Queiroz, and D. M. Dawson, Robust Adaptive Asymptotic Tracking of Nonlinear Systems With Additive Disturbance Louisiana State Univ., Baton Rouge, LA, Tech. Rep. ME-MS1-05, Jun. 2005.

[5] W. E. Dixon, Y. Fang, D. M. Dawson, and T. J. Flynn, "Range identification for perspective vision systems," IEEE Trans. Autom. Control, vol. 48, no. 12, pp. 2232-2238, Dec. 2003.

[6] S. Gupta, D. Aiken, G. Hu, and W. E. Dixon, "Lyapunov-based range and motion identification for a nonaffine perspective dynamic system," in Proc. IEEE Amer. Control Conf., Minneapolis, MN, 2006, pp. 4471-4476.

[7] H. K. Khalil, Nonlinear Systems, 3rd ed. Upper Saddle River, NJ: Prentice-Hall, 2002.

[8] M. Krstic, I. Kanellakopoulos, and P. Kokotovic, Nonlinear and Adaptive Control Design. New York: Wiley, 1995.

[9] F. L. Lewis, J. Campos, and R. Selmic, Neuro-Fuzzy Control of Industrial Systems With Actuator Nonlinearities. Philadelphia, PA: SIAM, 2002.

[10] F. L. Lewis, A. Yesildirek, and K. Liu, "Multilayer neural-net robot controller with guaranteed tracking performance," IEEE Trans. Neural Netw., vol. 7, no. 2, pp. 388-399, Mar. 1996.

[11] W. Lin and C. Qian, "Adaptive control of nonlinearly parameterized systems: A nonsmooth feedback framework," IEEE Trans. Autom. Control, vol. 47, no. 5, pp. 757-774, May 2002.

[12] M. Loffler, N. Costescu, and D. Dawson, "Qmotor 3.0 and the Qmotor robotic toolkit-An advanced PC-based real-time control platform," IEEE Control Syst. Mag., vol. 22, no. 3, pp. 12-26, Jun. 2002. 
[13] C. Makkar, "Nonlinear Modeling, Identification, and Compensation for Frictional Disturbances" M.S. thesis, Univ. of Florida, Gainesville, FL, 2006 [Online]. Available: http://etd.fcla.edu/UF/UFE0014262/ makkar_c.pdf

[14] C. Makkar, W. E. Dixon, W. G. Sawyer, and G. Hu, "Lyapunov-based tracking control in the presence of uncertain nonlinear parameterizable friction," in Proc. IEEE Amer. Control Conf., Portland, OR, 2005, pp. 1975-1980.

[15] C. Makkar, G. Hu, W. G. Sawyer, and W. E. Dixon, "Lyapunov-based tracking control in the presence of uncertain nonlinear parameterizable friction," IEEE Trans. Autom. Control, vol. 52, no. 10, pp. 1988-1994, Oct. 2007.

[16] C. Makkar, W. E. Dixon, W. G. Sawyer, and G. Hu, "A new continuously differentiable friction model for control systems design," in Proc. IEEE/ASME Int. Conf. Adv. Intell. Mechatronics, Monterey, CA, 2005, pp. 600-605.

[17] M. McIntyre, W. E. Dixon, D. M. Dawson, and I. D. Walker, "Fault detection and identification for robot manipulators," IEEE Trans. Robot., vol. 21, no. 5, pp. 1028-1034, Oct. 2005.

[18] P. M. Patre, W. MacKunis, C. Makkar, and W. E. Dixon, "Asymptotic tracking for systems with structured and unstructured uncertainties," in Proc. IEEE Conf. Decision Control, San Diego, CA, 2006, pp. 441-446.

[19] M. M. Polycarpou, "Stable adaptive neural control scheme for nonlinear systems," IEEE Trans. Autom. Control, vol. 41, no. 3, pp. 447-451, Mar. 1996.

[20] Z. Qu, Robust Control of Nonlinear Uncertain Systems. New York: Wiley, 1998
[21] S. Sastry and M. Bodson, Adaptive Control: Stability, Convergence, and Robustness. Upper Saddle River, NJ: Prentice-Hall, 1989.

[22] A. Serrani, A. Isidori, and L. Marconi, "Semiglobal nonlinear output regulation with adaptive internal model," IEEE Trans. Autom. Control, vol. 46, no. 8, pp. 1178-1194, Aug. 2001.

[23] J. J. Slotine and W. Li, Applied Nonlinear Control. Upper Saddle River: Prentice-Hall, 1991.

[24] V. I. Utkin, Sliding Modes in Control and Optimization. New York: Springer-Verlag, 1992.

[25] B. Xian, D. M. Dawson, M. S. de Queiroz, and J. Chen, "A continuous asymptotic tracking control strategy for uncertain multi-input nonlinear systems," IEEE Trans. Autom. Control, vol. 49, no. 7, pp. 1206-1211, Jul. 2004

[26] B. Xian, M. S. de Queiroz, and D. M. Dawson, A Continuous Control Mechanism for Uncertain Nonlinear Systems in Optimal Control, Stabilization, and Nonsmooth Analysis. Heidelberg, Germany: SpringerVerlag, 2004, pp. 251-262.

[27] B. Xian, M. S. de Queiroz, D. M. Dawson, and M. McIntyre, "A discontinuous output feedback controller and velocity observer for nonlinear mechanical systems," Automatica, vol. 40, no. 4, pp. 695-700, Apr. 2004.

[28] X. Zhang, A. Behal, D. M. Dawson, and B. Xian, "Output feedback control for a class of uncertain MIMO nonlinear systems with nonsymmetric input gain matrix," in Proc. IEEE Conf. Decision Control/ Eur. Control Conf., Spain, 2005, pp. 7762-7767. 